\title{
GIS-based Route Finding using Ant Colony Optimization and Urban Traffic Data from Different Sources
}

\author{
M. Davoodi ${ }^{\text {a, } *, \text { M. S. Mesgari }}{ }^{\text {b }}$ \\ ${ }^{a}$ Student of Geomatics Engineering, Faculty of Geodesy \& Geomatics Engineering, K. N. Toosi University of Technology - \\ Mojtaba.davoodi@ut.ac.ir \\ ${ }^{\text {b }}$ Faculty of Geodesy \&amp; Geomatics Engineering, K. N. Toosi University of Technology - mesgari@kntu.ac.ir
}

Commission VI, WG VI/4

KEY WORDS: Route Finding, Ant Colony Optimization, Urban Traffic Data, Floating Car Data, Video Vehicle Detection

\begin{abstract}
:
Nowadays traffic data is obtained from multiple sources including GPS, Video Vehicle Detectors (VVD), Automatic Number Plate Recognition (ANPR), Floating Car Data (FCD), VANETs, etc. All such data can be used for route finding. This paper proposes a model for finding the optimum route based on the integration of traffic data from different sources. Ant Colony Optimization is applied in this paper because the concept of this method (movement of ants in a network) is similar to urban road network and movements of cars. The results indicate that this model is capable of incorporating data from different sources, which may even be inconsistent.
\end{abstract}

\section{INTRODUCTION}

Route finding is a vital network analysis which is the basis of some other GIS-based analysis. Ant colony algorithm is proposed by Marco Dorigo in 1992 for finding the shortest path in a graph. ACO could be considered as a suitable algorithm for route finding because it symbolizes the movement of ants in a graph, marked by a substance called pheromone. In this paper a novel model based on ACO is proposed to find the shortest path using traffic data from different sources. In the first step, traffic data from buses which are equipped with GPS is collected to initialize the pheromone values. In other words, pheromone in each edge is related to the average speed of the bus in this edge. Therefore, in opposition to the ordinary ACO, here the pheromone is not zero in the beginning of the process. Following that, the traffic data from VVD is used to update pheromone values in each edge. The main important contribution of this research is that this method can find the optimum path much faster than other algorithms because it benefits from initializing the pheromone values from FCD traffic data.

\section{METHODS AND MATERIALS}

\subsection{Ant Colony Optimization}

"ACO is a meta-heuristic technique that uses artificial ants to find solutions to combinatorial optimization problems. ACO is based on the behaviour of real ants searching a path between their colony and a source of food" (Claes, et al., 2011) (See Figure 1)

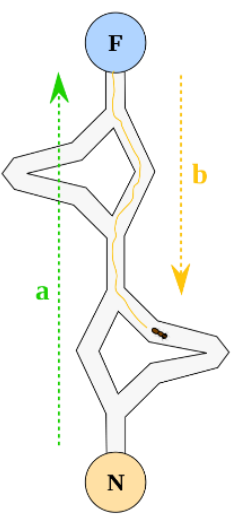

1

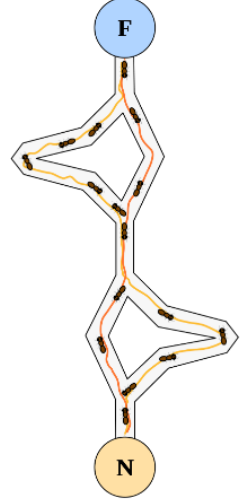

2

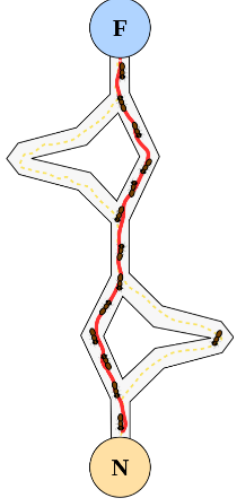

3
Figure 1. Behaviour of real ants

In the real world ants move around their colony and search for food and release a chemical substance named "Pheromone". This substance attracts other ants, so when a food be found somewhere, the ants follow the first ant that found the food and more ants attracts to the food and that route be desired one; so many ants move on that route.

This behavior can be simulated in computational problems. Some initial answers can be considered, then in an iteration a positive score added to good answers and bad answers have been deleted. This process will continued until best answer be found.

There is an important step in this process called "pheromone update" which mentioned above. In each step a positive score will be added to all answers, so good answers get more pheromone than other answers, so the good answers risen and at the end of iteration the best answer will be found. Also these pheromones will not remain permanently and they will disappear slowly. This process is evaporation and there is a parameter in ACO algorithm that called "evaporation rate".

Because of the nature of route finding problem, it's very similar to ant's movements; so the Ant Colony Optimization algorithm is very useful in route finding. A route includes some nodes (like

\footnotetext{
* Corresponding author
} 
$\mathrm{i}, \mathrm{j} . .$.$) and edges. In simple ACO the value of an edge$ corresponded with its length. The pheromones considered for each edge as a value. Several routes between origin and destination will considered and evaluated. Better solutions attracts more pheromones in pheromone update process so that at the end of iteration the best route attracts most pheromone and introduced as best solution between two points. And then the shortest path between two points will be found. (See Figure 2) Pheromone update equation is:

$$
\tau_{i j} \leftarrow(1-\rho) . \tau_{i j}+\sum_{k=1}^{m} \Delta \tau^{k}{ }_{i j}
$$

Where $\rho$ is evaporation rate, $\mathrm{m}$ is number of ants.

$$
\Delta \tau_{i j}^{k}=\left\{\begin{array}{rr}
\frac{Q}{L_{k}} & \text { if ant } k \text { used edge }(i, j) \text { in its route } \\
0 & \text { otherwise }
\end{array}\right.
$$

Where $\mathrm{Q}$ is a constant, $L_{k}$ is route length of $\mathrm{k}$-th ant.

The probability $p^{k}{ }_{i j}$ of the $\mathrm{k}$-th ant moving from point $\mathrm{i}$ to $\mathrm{j}$ is:

$p^{k}{ }_{i j}=\left\{\begin{array}{lr}\frac{\tau^{\alpha}{ }_{i j} \cdot \tau^{\beta}{ }_{i j}}{\sum_{l \in \text { allowed }_{k} \tau^{\alpha}{ }_{i l} \cdot \eta^{\beta}{ }_{i l}}} & \text { if } j \in \text { allowed }_{k} \\ 0 & \text { otherwise, }\end{array}\right.$

Where allowed $_{k}$ is the list of nodes not yet visited by $k$-th ant But a shortest path between two points isn't always good choice. Because the ACO algorithm only considers the length of the route and it may be a narrow street or busy road. So the selected route between origin and destination may have heavy traffic.

So there is another parameter can be considered and used as a value in pheromone update process. In this paper we initialize the pheromones with bus speed which collected from FCD method and when some answers considered as initial answers, the pheromones updated with data from Video Vehicle Detection method; so the road traffic have been considered and travel time decreased. The FCD and VVD methods described in next section.

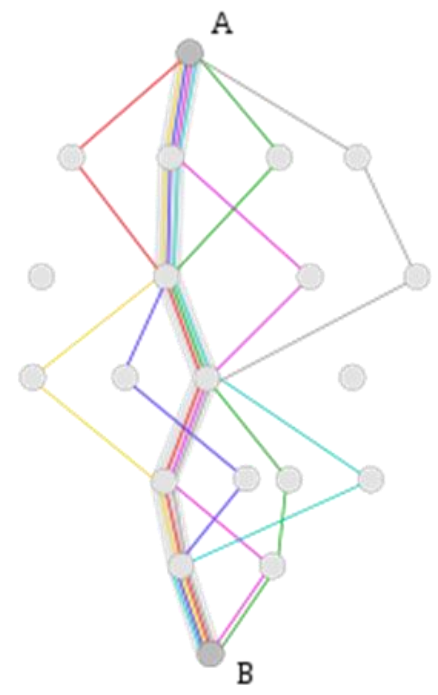

Figure 2. Shortest path using ACO

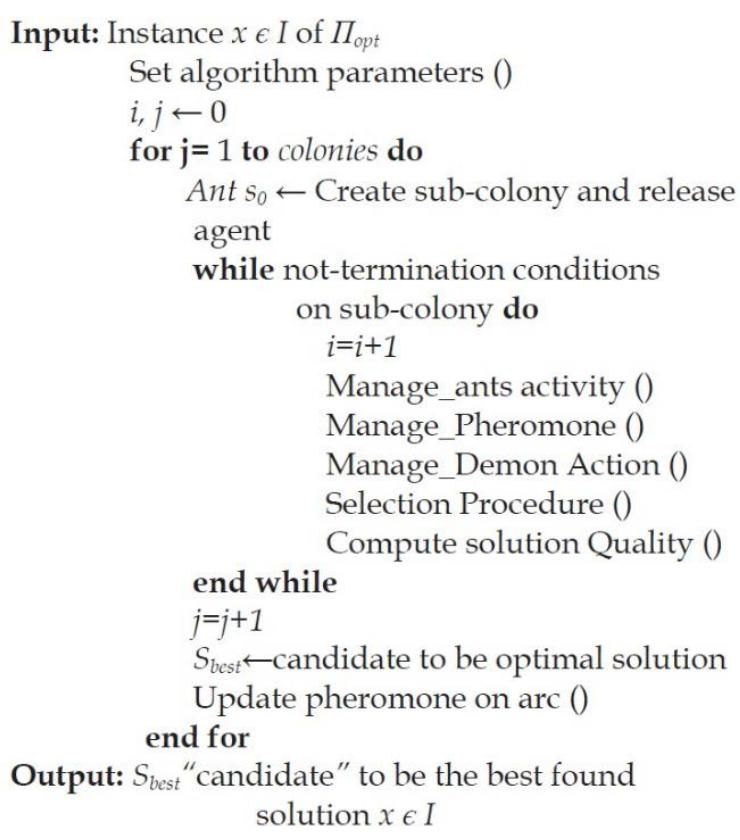

Figure 3. ACO pseudo Code

\subsection{Traffic Data Collection Methods}

2.2.1 Floating Car Data: FCD systems are used in several important applications since these systems resolve the limitations of fixed traffic controlling methods (installation and maintenance costs, lack of flexibility, etc.) (Bishop, 2005).

Floating Car Data is a method so as to determine the velocity of the traffic of roads. This method is based on collecting data of location, velocity, direction, and time information; which are derived from mobile devices. These data are an important source for traffic information, and they are used much more in Intelligent Transportation Systems (ITS). In this case, every automobile with a mobile device can act as a sensor for road network. Based on the data extracted in this manner, traffic jams can be determined, time travel can be computed, and traffic reports can be produced. In contrast to traffic cameras' methods, vehicle license plate recognition systems, and installed induction loops in roads, there is needed any other additional hardware on the road networks.

Floating Cellular Data is one of the methods for collecting traffic data. This method uses data from cellular networks (GSM and GPRS). Every mobile devices would become to a traffic sensor, and it is such an unknown information source. The Location of a mobile device can be calculated by two methods: 1) use triangulation techniques, 2) use of stored hand-over data by operator of the network. Because of having less accuracy that GSM locating has than systems based on GPS, many mobile devices must be tracked and must be used complicated algorithm in order to extracting well-quality data. In urban regions where traffic data often needed, the distances between telecommunication antennas is fewer. Therefore, with oldfashioned methods such as GPS sensors or video cameras, the precision will be improved. The advantages of this method over the methods which are based on GPS, or such older methods as traffic cameras, embedded sensors in streets, and to name but a few, there is no need to infrastructure or special hardware in automobiles or along roads. Besides that, it not only has a lower cost and a broaden coverage, but also need lower time for configuration and maintenance. 
Car Recognition: Car recognition methods need a group of detectors settled along the road. In this technique, a unique serial number recognize for a car in a location and in another location repeat again. (Recognition) Travel time and velocity are identified and computed by a comparison between the times at which a car passed through two sensors. Hence, the order of traffic information is produced.

For instance, WISERIDE - www.wiseride.gr - is a traffic information system, which is like routing tools, based on FCD. The derived location and velocity data from GPS device installed in taxies formulate the map of all over a city (WISERIDE, 2015). There is another system that exactly called "Floating Car Data", in this method some distinguished cars equipped with several sensors like humidity sensor, pressure sensor, temperature sensor, rain sensor, speed sensor, crash sensor, distance detection, navigation (GPS) and suitable communication tools to send these information to traffic control center (See Figure 4).

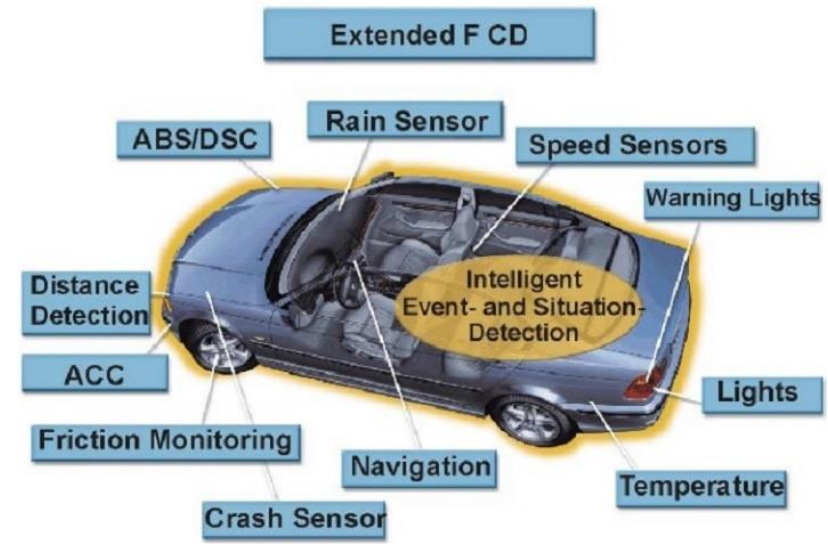

Figure 4. Embedded sensors in floating cars

These cars move in city streets and get in traffic flow. So a floating car moves in each street with other cars and collects traffic information like traffic flow mean speed, temperature, pressure, humidity, ... which traffic flow mean speed is the most important one that describes traffic situation of that street. Different decision making processes can be done by sending these information to traffic control center.

Useful information about situation of city streets can be produced as it illustrated in Figure 5. For example if an accident occurs in a street, this car can report to traffic control center; then this information quickly can be send to another car drivers that want to enter this street and help them (They can go to another street). Also meteorological information like frost route or fog are example of this useful information.

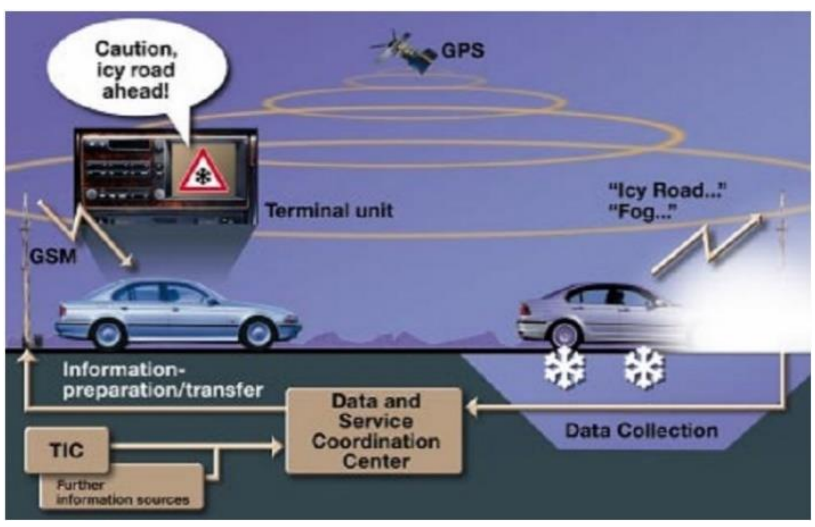

Figure 5. Different produced information by floating cars
Also sometimes there need be two or three floating car in a street because it may be different traffic situation between beginning and end of a street! (See Figure 6) If there is just one floating car in the beginning of the street it report high mean speed while mean speed of traffic is low at the end of street. So it's better to have more than 2 floating car in each street.

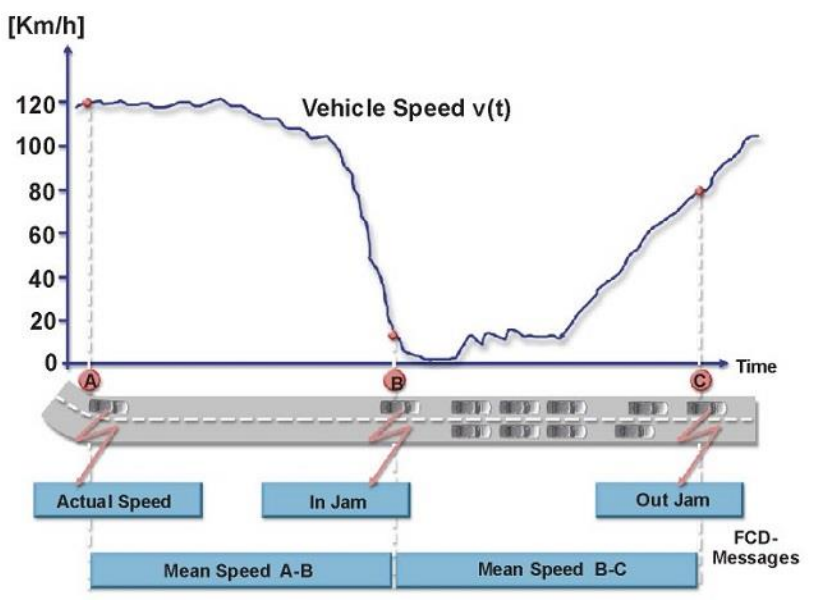

Figure 6. Different traffic speed in one street

2.2.2 Video Vehicle Detection: Video Vehicle Detection recently is becoming the most common method to replace the old technologies like loop detectors for the detection of vehicles in road networks (Rahmat, et al., 2000).

Video Vehicle Detection cameras work based on Image Processing algorithms and detect cars; so they provide traffic detection, number and speed of vehicles and also detecting stopped cars (Middleton, et al,. 2004). (See Figure 7) The cameras installed on the top or near the roads. Most of these systems need initial settings including elevation of camera in the road and distance between lanes. But system performance decrease in night and we need to install many cameras in all streets to gain all the traffic information of whole city.

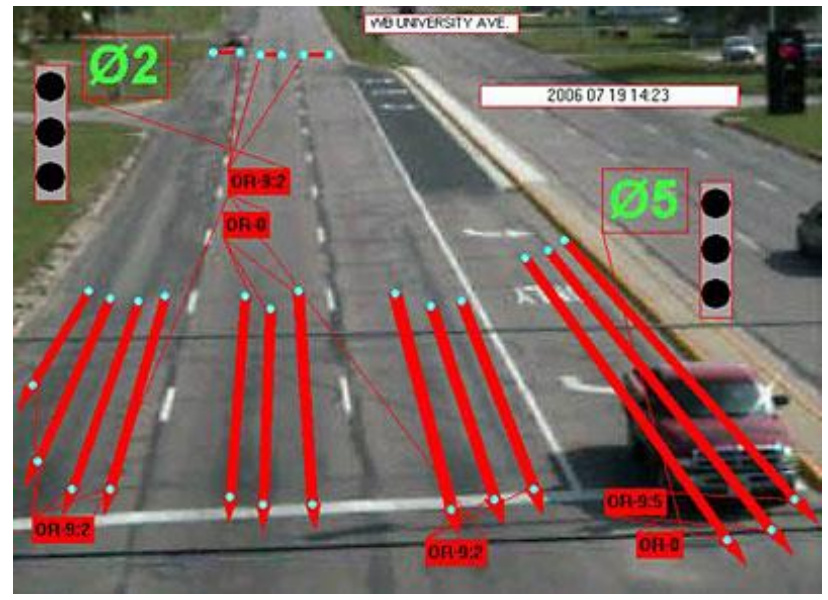

Figure 7. A video image frame analyzing

\section{RESULTS}

The two routes imported to ArcGIS software and drawn. The results of the proposed model are illustrated in Figure 8 which shows an optimum path between the University of Tehran and K.N.Toosi University of Technology. Figure 9 depicts the 
The International Archives of the Photogrammetry, Remote Sensing and Spatial Information Sciences, Volume XL-1/W5, 2015 International Conference on Sensors \& Models in Remote Sensing \& Photogrammetry, 23-25 Nov 2015, Kish Island, Iran

convergence diagram of the proposed model. It can be concluded from Figure 3 that initializing the pheromones with FCD method can substantially reduce the convergence time. Therefore, it takes much less time to find the optimum path in a mega city.

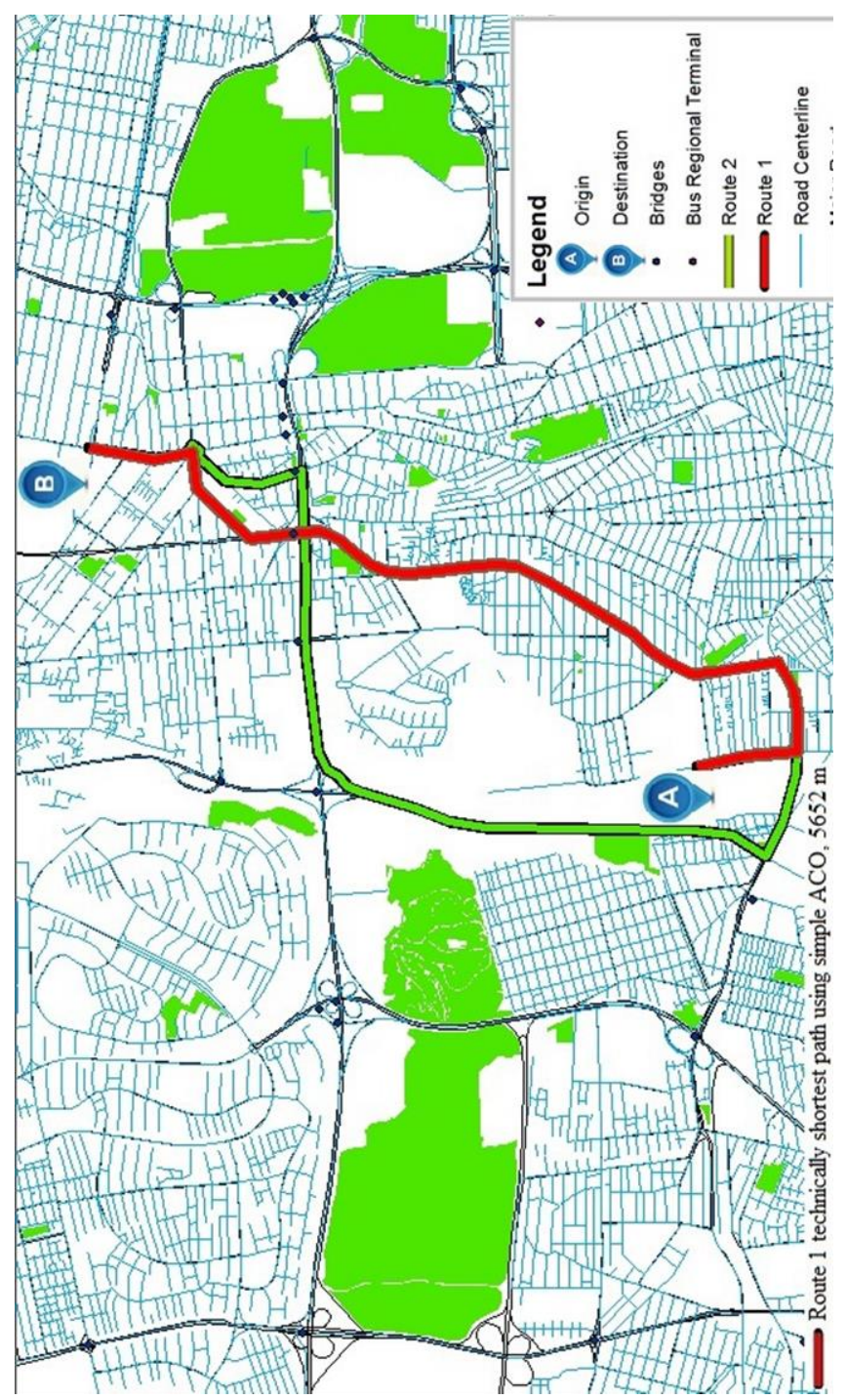

Figure 8. Optimum path between point A and B

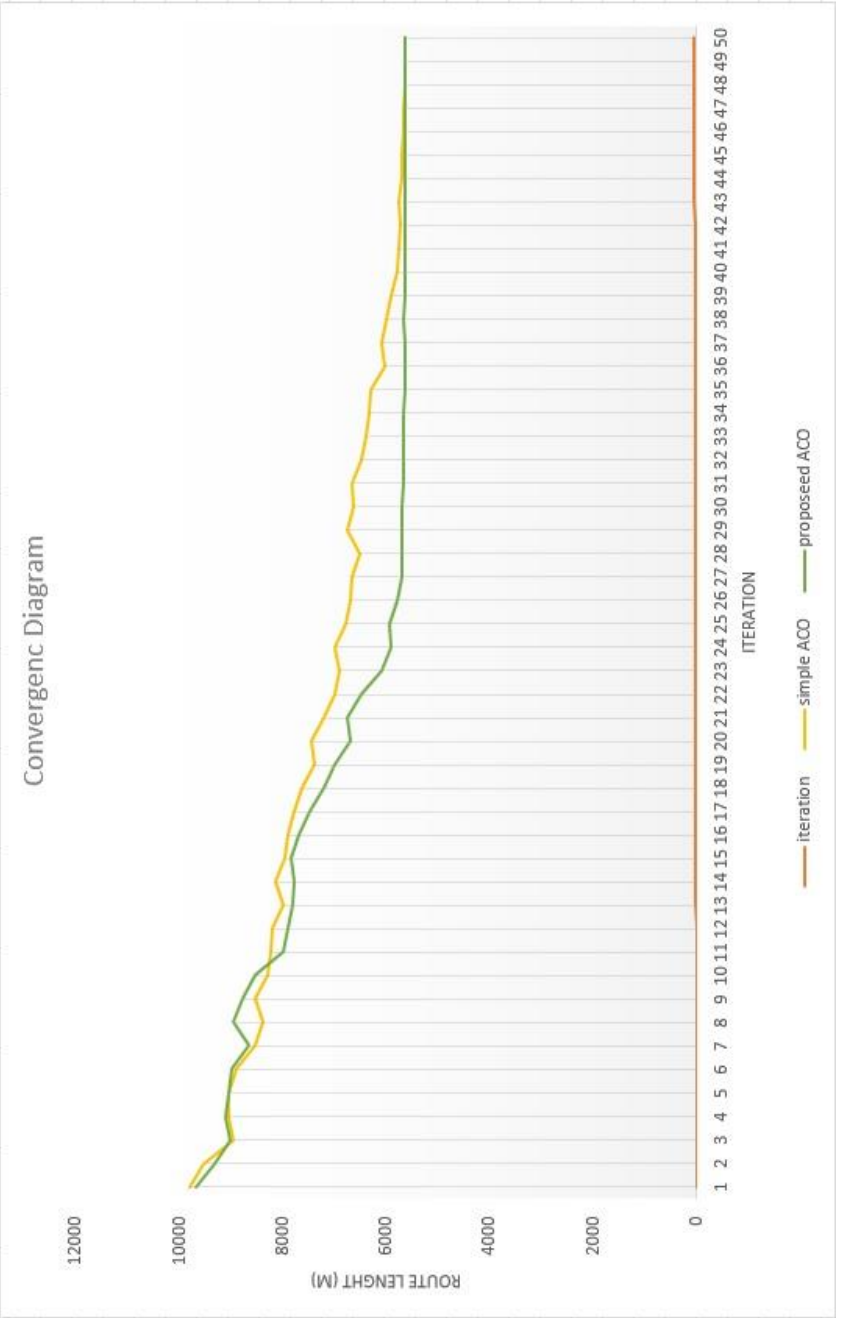

Figure 9. Convergence diagram of the proposed model

\section{CONCLUSION}

A novel route finding model is proposed in this paper based on the integration of traffic data collected from FCD and VVD. Ant colony optimization which is able to find the shortest route in a given graph is used. Pheromone values are initialized with the data from FCD method (buses equipped with GPS receivers). This data provides us with a rough understanding about traffic condition. Initializing ACO pheromone values with FCD data can dramatically reduce the number of iterations needed for convergence of the algorithm. Therefore, the proposed model is substantially faster than simple ACO.

\section{REFERENCES}

$\mathrm{R}$ Bishop, Intelligent Vehicle Technologies and Trends (Artech House, Boston, Mass, USA, 2005).

Rutger Claes, Tom Holvoet. Ant Colony Optimization applied to Route Planning using Link Travel Time predictions. IEEE International Parallel \& Distributed Processing symposium. 2011 May; 358-365. 
The International Archives of the Photogrammetry, Remote Sensing and Spatial Information Sciences, Volume XL-1/W5, 2015 International Conference on Sensors \& Models in Remote Sensing \& Photogrammetry, 23-25 Nov 2015, Kish Island, Iran

Middleton, D. White, R. Crawford, J. Song, J. Haas, "Initial Investigation for Traffic Monitoring Equipment Evaluation Facility," Research Report 0-4664-1, Texas Transportation Institute, College Station, TX, 2004.

R. A. Rahmat, K. Jumari, 2000: Vehicle Detection Using Image Processing For Traffic Control And Surveillance System. Proceeding of 7th World Congress on Intelligent Transport Systems.

Wiseride [Internet]. [cited 2015 Oct 15]. Available from: http://www.wiseride.gr/ 